\title{
Multichannel Blind Deconvolution in Eye Fundus Imaging
}

\author{
Andrés G. Marrugo*, Michal Šorel ${ }^{\dagger}$, Filip Šroubek ${ }^{\dagger}$ and María S. Millán* \\ ${ }^{*}$ Group of Applied Optics and Image Processing \\ Dept. of Optics and Optometry, Universitat Politècnica de Catalunya \\ Violinista Vellsolà 37, 08222 Terrassa, Spain. \\ Email: andres.marrugo@upc.edu \\ ${ }^{\dagger}$ Institute of Information Theory and Automation \\ Academy of Sciences of the Czech Republic \\ Pod Vodárenskou věží 4, 18208 Prague 8, Czech Republic.
}

\begin{abstract}
Eye fundus imaging is vital for modern ophthalmology. Due to the acquisition process, fundus images often suffer from blurring and uneven illumination. This hinders diagnosis and the evolution assessment of a disease. We present a method for fundus image deblurring by means of multichannel blind deconvolution. It consists of a series of preprocessing steps to adjust the images so they comply with the considered degradation model, followed by the estimation of the point spread function, and image deconvolution. Results show that our approach is capable of significant resolution improvement in degraded retinal images.
\end{abstract}

\section{INTRODUCTION}

Digital imaging of the eye fundus is widely used to diagnose and manage ophthalmologic disorders such as diabetic retinopathy and age-related macular degeneration [1]. Fundus images are captured using a conventional digital camera, attached to a retinal camera body designed to image the eye fundus in association with the optical system of the eye. Major source of retinal image quality degradation are aberrations of the eye, imperfections in the fundus camera optics, and improper camera adjustment. The imaging procedure is usually carried in two separate steps: Image acquisition and diagnostic interpretation. Image quality is subjectively evaluated by the person capturing the images and they can sometimes mistakenly accept a low quality image. A recent study by Abràmoff et al. [2] using an automated system for detection of diabetic retinopathy found that from 10000 exams $23 \%$ had insufficient image quality.

In this paper we develop a practical strategy for retinal image deblurring. The core of our proposal is based on an image processing technique for restoration called blind deconvolution (BD) [3]. The goal of BD is to recover the original scene from a single or set of blurred images in the presence of a poorly determined or unknown point spread function (PSF). The main assumption is that blur can be described by a convolution of a sharp image with the unknown PSF. There are basically two groups of BD algorithms; one group that uses a single input image (single-image blind deconvolution SBD) and the other multiple images of the same object blurred in a different way (multichannel blind deconvolution MBD). There are many reliable SBD algorithms [4], however most of them require that the blurred image be governed by relatively strong edges, which is not case here. Therefore we have chosen MBD as a suitable strategy for the restoration of blurred retinal images.

The paper is organized in the following way. Initially we describe a general model for the image degradation which encompasses blurring and uneven illumination, subsequently we detail our approach which consists in an extensive preprocessing stage followed by the PSF estimation and image deconvolution. Finally we discuss a result of the image enhancement strategy and compare with a recent state-of-the-art SBD method [5].

\section{Mathematical Model of image Degradation}

We assume two registered input images, $z_{1}$ and $z_{2}$, both originating from an ideal sharp image $u$

$$
\begin{aligned}
& z_{1}=u * h_{1}+n_{1} \\
& z_{2}=\left(u k^{-1}\right) * h_{2}+n_{2},
\end{aligned}
$$

where $*$ is the standard convolution, $h_{i}$ are called convolution kernels or point-spread functions (PSFs) and $k$ is a function accounting for relative local illumination change between images $z_{1}$ and $z_{2}$. For pixels where no illumination changes occur $k \approx 1$. The noise $n_{i}$ is assumed Gaussian additive with zero mean in both images. In our case, the PSFs comprise all radiometric degradations described above except structural changes in the eye. Despite the fact that we consider the PSFs to vary in time between the two image acquisitions, we assume them to be spatially invariant within each image.

\section{Algorithm}

In the input, the algorithm accepts two eye fundus images. The images are processed in the following four steps:

1) Image registration

2) Compensation of uneven illumination

3) Segmentation of areas with structural changes

4) PSF estimation and Image deconvolution

The individual steps are detailed in the following subsections. For illustration purposes we consider two color fundus images acquired from a patient that suffered from age-related macular 


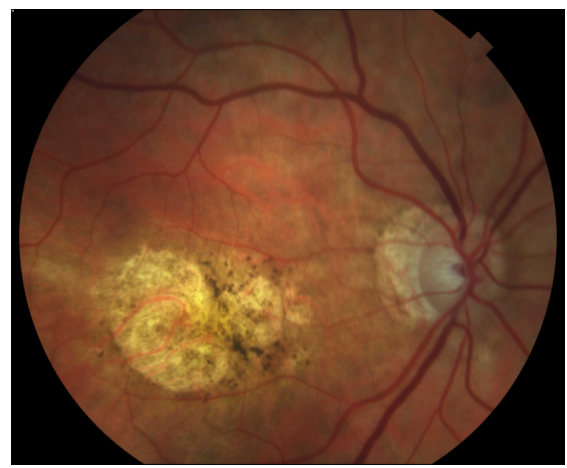

(a) $z_{1}$

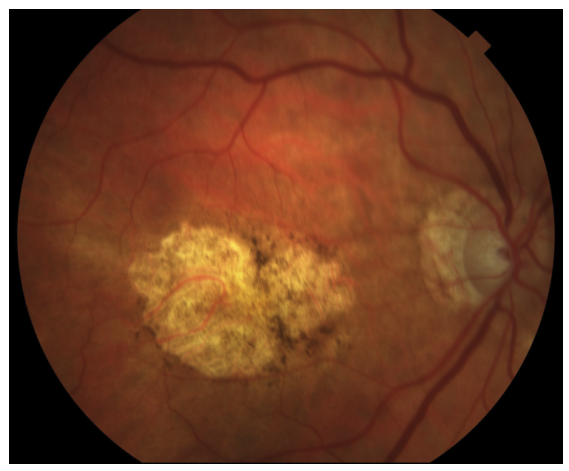

(b) $z_{2}$

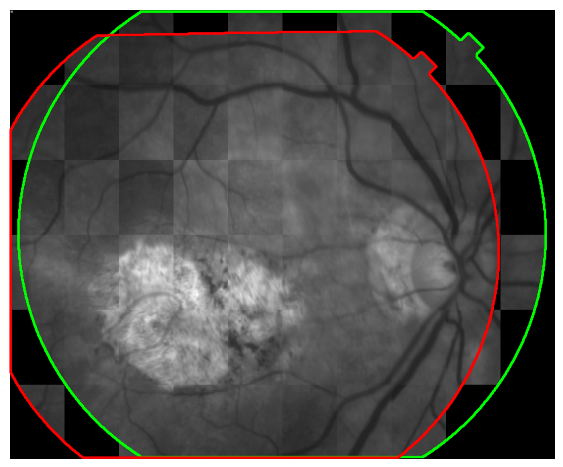

(c)

Fig. 1. Color eye fundus images affected by age-related macular degeneration. (a) Image $z_{1}$ was captured seven months prior to (b) image $z_{2}$. (c) Registration of images $z_{1}$ and $z_{2}$ in checkerboard representation.

degeneration, which we denote hereafter by $z_{1}$ and $z_{2}$ and their enhanced versions as $u_{1}$ and $u_{2}$. The images were captured seven months apart from each other and are shown in Fig. 1. They are color 24 bit-depth fundus images of size $1500 \times 1230$ digitized in TIFF format. This is a general example were both images do not correspond exactly to the same object field, the illumination distribution across both images is not exactly the same, and there are some structural differences between them given by the pathological development in the macula (centered yellowish region).

\section{A. Image registration}

Image registration consists in the spatial alignment of two or more images. Image registration techniques are usually divided into two groups: intensity-based and feature-based methods [6]. Intensity based methods have the drawback of poor performance under varying illumination conditions. Feature based methods are robust to such effects but rely on accurate and repeatable extraction of the features. The retinal vasculature is known to provide a stable set of features for registration for the conditions of interest.

For registering the images we use the robust dual-bootstrap iterative closest point algorithm [7]. The vessel branching and crossover points are used as landmarks to register the images. The registration algorithm starts from initial low-order estimates that are accurate only in small image regions called bootstrap regions. The transformation is then refined using constraints in the region, and the bootstrap region is expanded iteratively. The algorithm stops when the boot-strap region expands to cover the overlap between the images, and uses a 12-dimensional quadratic mapping that accounts for the curvature of the retina. This registration algorithm is very robust to local changes and low overlap between images as demonstrated by its high success rate on test images with at least one common landmark point and overlaps even as low as $35 \%$ [7]. The pair of images after registration are shown in Fig. 1(c) in checkerboard representation. For the following subsection, we will consider only the overlapping area of both registered fundus images as a region of interest (ROI).

\section{B. Compensation of uneven illumination}

Despite controlled conditions in retinal image acquisition, there are many patient-dependent aspects that are difficult to control and mainly affect the illumination component with gradual non-uniform spatial variations [1]. Some of the contributing factors are: (a) the curved surface of the retina. As a consequence, all regions cannot be illuminated uniformly; (b) Imaging requires either a naturally or an artificially dilated pupil. However, the degree of dilation is highly variable across patients; (c) Unexpected movements of the patients eye and (d) presence of diseases. This effect hinders both quantitative image analysis and the reliable operation of subsequent global operators.

In our model, described by (1), the relative changes in intensity between two fundus images of the same eye cannot be accounted exclusively by convolution with different PSFs and must be compensated. A number of general-purpose techniques have been investigated to attenuate the variation of illumination. However, most techniques, ranging from simple histogram operations to more elaborate models, are oriented towards single-image compensation [1]. Thus, no consistency between a pair of images is guaranteed. This uneven illumination can be compensated by adjusting the intensity values on one image to approximately match that of the other; i.e. we simply compensate the relative changes of illumination between the images so they meet the requirements from the model. This can be carried out if the blurring is not too large and the illumination changes smoothly, which is usually the case for eye fundus images. 


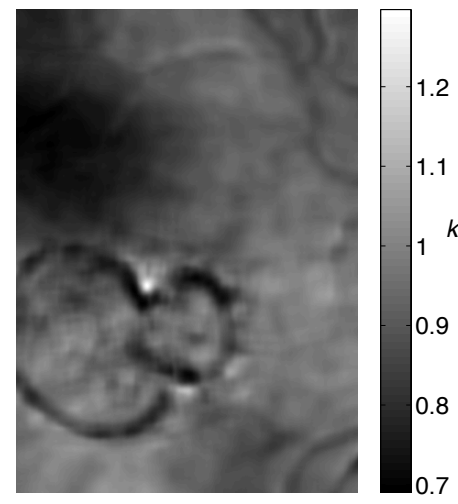

(a)

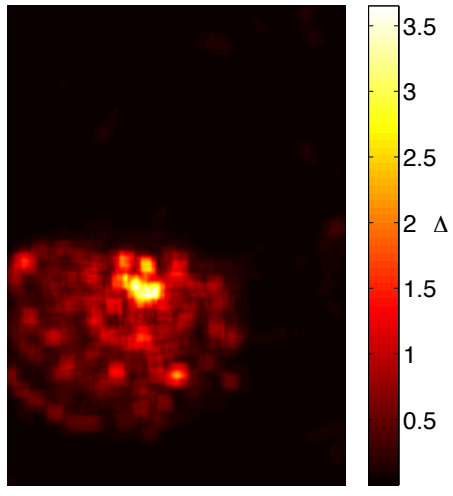

(b)

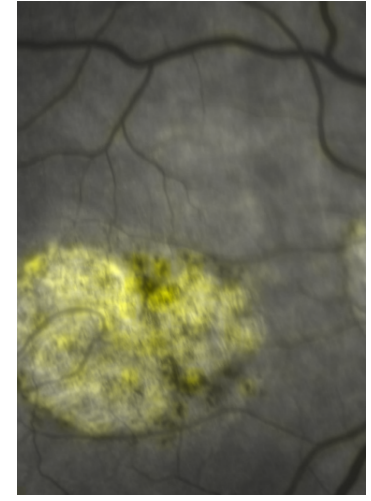

(c)

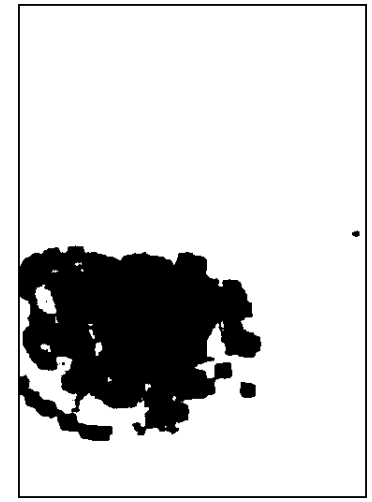

(d)

Fig. 2. Intermediate outputs from the algorithm: (a) illumination compensation function $k$, (b) local quadratic difference $\Delta$, (c) areas of structural change, and (d) mask for avoiding areas with structural changes.

For the illumination compensation we use a sliding window procedure. This provides a simple and effective way to estimate the smooth variation of illumination [8], [9]. To get the compensation function $k$ of our model (1), in the square neighborhood of fixed width $W^{j}$ of each pixel $j$ we minimize

$$
k^{j}=\arg \min _{\kappa} \sum_{i \in W^{j}}\left(z_{1}^{i}-\kappa z_{2}^{i}\right)^{2},
$$

where $z_{1}$ and $z_{2}$ are the registered input images. By differentiating (2) with respect to $\kappa$ we obtain the expression for $k^{j}$ :

$$
k^{j}=\frac{\sum_{i \in W^{j}} z_{1}^{i} z_{2}^{i}}{\sum_{i \in W^{j}} z_{2}^{i^{2}}} .
$$

The interpretation of $k$ from (2) is straightforward. If the registered images $z_{1}$ and $z_{2}$ had neither illumination changes nor structural changes, then $k \approx 1$ throughout the common object field. The reason why the computation by the sliding window works is that illumination changes in fundus images are smooth and can be considered locally constant, together with the fact that convolution does not change the total image energy. Since the sums in (3) can be computed in an incremental way, the computation of $k$ is very fast, with time complexity proportional to the number of pixels.

The resulting $k$ computed with a window 17 pixels wide is shown in Fig. 2(a). The different shades of gray indicate the average contrast and intensity difference between the two images. From the image it can be seen that most areas have similar intensity values except for the upper left part (dark region), where image $z_{2}$ is up to 30 percent darker. This is also noticeable for the bottom-left quadrant (the pathological damaged region) where the differences are not due to illumination variation, but to morphological or structural changes, thus the values have no meaning in terms of illumination compensation.

\section{Segmentation of areas with structural changes}

The pathological region is actually a structural change and cannot be taken as illumination variation. To identify these changes we computed the local quadratic difference from the two images including illumination compensation as:

$$
\Delta^{j}=\sum_{i \in W^{j}}\left|z_{1}^{i}-k z_{2}^{i}\right|^{2}
$$

We highlight the fact that structural changes can be located with this approach because the illumination varies smoothly, whereas structural changes are often local and appear abruptly. The interpretation of (4) is rather simple, the output is close to zero where the illumination has been adequately compensated and the opposite in areas where the images differ structurally. The result, shown in Fig. 2(b), clearly reveals the existence of regions that after illumination compensation still differ significantly. If these regions appear clustered in the image, one suspects they are probably caused by a source of variation that is not related to illumination variation.These are most likely related to pathological damage which is significant from the clinical viewpoint. To better understand this result, in Fig. 2(c) we show one of the retinal images in gray-scale where the pixels related to structural changes are represented in pseudo-color. This image constitutes an important output of our algorithm. On the other hand, since these changes do not fulfill our convolution model, they should be masked out in order to correctly estimate the PSFs from both images. [10].

In our experiments, we applied Otsu's thresholding method [10] to automatically generate a mask from the difference image $\Delta$ (Fig. 2(b)). In this way the regions that have structurally changed are are not included in the minimization routine. This is not critical because in practice there is enough information in the remaining pixels to adequately estimate the PSFs. The obtained mask is shown in Fig. 2(d).

\section{PSF estimation and image deconvolution}

For PSF estimation and image deconvolution we have chosen one of the best working MBD methods [12]. Matlab implementation of this method is available on the web of the authors ${ }^{1}$. The algorithm can be viewed as a Bayesian maxi-

\footnotetext{
${ }^{1}$ http://zoi.utia.cas.cz/download
} 
mum a posteriori estimation of the most probable sharp image and blur kernels. For our purposes, we used a modification of the original method that ignores regions affected by structural changes, which improves stability and precision of the computation. This is similar to the solution proposed in [13] within the super-resolution context. Without this modification, represented by the mask $m$ in (5), the algorithm does not work reliably. The algorithm can be described as a minimization of the functional:

$$
\begin{array}{r}
\arg \min _{u, h_{1}, h_{2}} \frac{1}{2}\left\|u * h_{1}-z_{1}\right\|^{2}+\frac{1}{2}\left\|m\left(u * h_{2}-k z_{2}\right)\right\|^{2} \\
+\lambda_{u} \int|\nabla u|+\lambda_{h}\left\|m\left(z_{1} * h_{2}-k z_{2} * h_{1}\right)\right\|^{2}, \\
h_{1}, h_{2} \geq 0,
\end{array}
$$

with respect to the latent image $u$ and blur kernels $h_{1}$ and $h_{2}$. The first and second terms measure the difference between the input blurred images and the searched image $u$ blurred by kernels $h_{1}$ and $h_{2}$. The size of this difference is measured by $L_{2}$ norm $\|$.$\| and should be small for the correct solution.$ Ideally, it should correspond to the noise variance in the given image. Function $k$ compensates for uneven illumination, as described in Section III-B. The value of the masking function $m$ is one in the valid points (white in Fig. 2(d)) and zero in the pixels where the eye fundus is significantly different; in these pixels, we consider only the information from image $z_{1}$. The two remaining terms are regularization terms with positive weighting constants $\lambda_{u}$ and $\lambda_{h}$. The third term is nothing else than the total variation of image $u$. It improves stability of the minimization and from the statistical viewpoint incorporates prior knowledge about the solution. The last term is a condition linking the PSFs of both images, which also improves the numerical stability of the minimization. For this procedure we set $\lambda_{u}=1000$ and $\lambda_{h}=10$.

The functional is alternately minimized in the subspaces corresponding to the image and the PSFs. The minimization in the PSF subspace is equivalent to the solution of a system of linear equations in the least squares sense with the non-negativity constraint (in our implementation solved by Matlab fmincon function). The deconvolution realized by the minimization in the image subspace, is solved by halfquadratic iterative scheme [14], replacing the total variation by $\int \sqrt{|\nabla u|^{2}+\epsilon^{2}}$ in order to achieve smoothness of the functional for zero gradients.

The image deconvolution, that is the procedure to obtain the enhanced versions of $z_{1}$ and $z_{2}$ consists in the following. The minimization of (5) yields $u_{1}, h_{1}$, and $h_{2}$. However, $u_{2}$ (the enhanced version of $z_{2}$ ) is obtained by minimizing (5) once more with fixed PSFs and masking $z_{1}$ instead of $z_{2}$. The enhanced RGB fundus image is obtained by estimating the PSF from the green channel (being the one with highest contrast) and subsequently deconvolving each channel independently.

\section{RESULTS}

The ROIs for each image $z_{1}$ and $z_{2}$, which consist of pathological area plus the unaltered surrounding area and blood vessels, are shown in Fig. 3. Both images are slightly blurred which hinders the possibility to properly resolve details. The PSFs estimated from the minimization of (5) are shown in figures 3(b) and 3(d). The difference in size of the PSFs indicates that one image is more blurred than the other. In Fig. 4(b) we show the enhanced version of $z_{2}$ by our method. There is a significant improvement in resolution evidenced by the clear definition of the thinner blood vessels and the small pathological structures in the center. To corroborate our assumption that MBD methods are better suited for this type of images, we tried to restore $z_{2}$ with a recent SBD method proposed in [5]. The result is shown in Fig. 4(a) and reveals that this method does not follow the true nature of the blurring, thus it is prone to produce a poor restoration. None of the aforementioned fine structures are properly resolved in this image. Finally, we show the color restored versions of $z_{1}$ and $z_{2}$ in Fig. 5. The enhancement is much more noticeable in color.

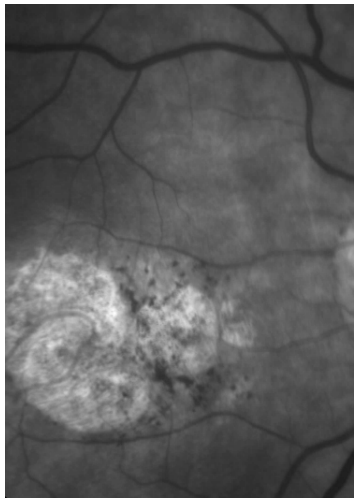

(a) ROI from $z_{1}$

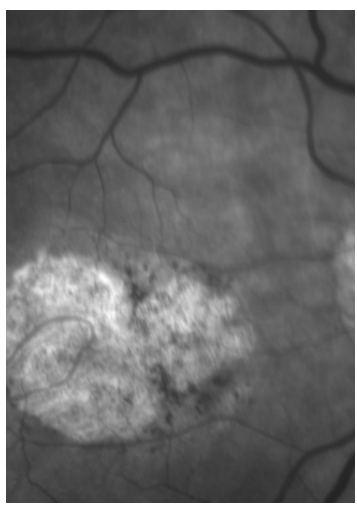

(c) ROI from $z_{2}$

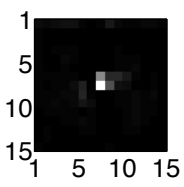

(b)

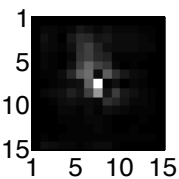

(d)
Fig. 3. ROIs of retinal images (a) $z_{1}$ and (c) $z_{2}$, and their corresponding estimated PSFs in (b) and (d), respectively. The enhanced version of $z_{2}$ is shown in Fig. 4(b).

\section{COnClusions}

In this paper we have presented a new approach for eye fundus image deblurring based on MBD. We have verified, that a pair of retinal images, belonging to the same eye, contain enough common information to be restored with the proposed method. The method consists of a series of 


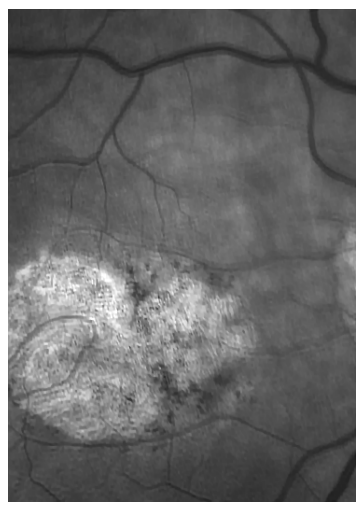

(a)

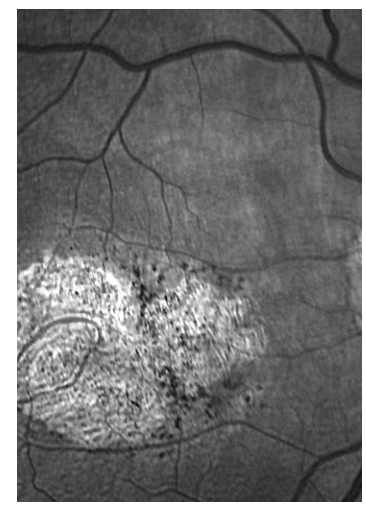

(b)
Fig. 4. Enhanced version of $z_{2}$ with (a) SBD method of [5] and (b) proposed method. There is a significant resolution improvement in (b), compare with the original in Fig. 3(c).

preprocessing steps to adjust the images so they comply with the convolutional model, followed by the final stages of PSF estimation and deconvolution. This approach leads to the improvement in resolution of degraded retinal images, which we have also compared with one of the most sophisticated SBD algorithms [5]. While the initial findings are promising, further tests are necessary.

\section{ACKNOWLEDGMENT}

This research has been partly funded by the Spanish Ministerio de Ciencia e Innovación y Fondos FEDER (project DPI2009-08879). Financial support was also provided by the Czech Ministry of Education under the project $1 \mathrm{M} 0572$ (Research Center DAR). Authors are grateful to the ophthalmologist Jordi Monés (MD) from the Institut de la Màcula i la Retina (Barcelona) for providing us the images. The first author also thanks the Spanish Ministerio de Educación for an FPU doctoral scholarship.

\section{REFERENCES}

[1] R. Winder, P. Morrow, I. McRitchie, J. Bailie, and P. Hart, "Algorithms for digital image processing in diabetic retinopathy," Comput Med Imag Grap, vol. 33, no. 8, pp. 608-622, 2009.

[2] M. D. Abràmoff, M. Niemeijer, M. S. A. Suttorp-Schulten, M. A. Viergever, S. R. Russell, and B. van Ginneken, "Evaluation of a system for automatic detection of diabetic retinopathy from color fundus photographs in a large population of patients with diabetes," Diabetes Care, vol. 31, no. 2, pp. 193-198, 2008.

[3] E. P. Campisi and K. Egiazarian, Blind Image Deconvolution: Theory and Applications. CRC Press, 1996.

[4] A. Levin, Y. Weiss, F. Durand, and W. Freeman, "Understanding and evaluating blind deconvolution algorithms," in Proc. IEEE Conf. Computer Vision and Pattern Recognition, 2009, pp. 1964 -1971.

[5] L. Xu and J. Jia, "Two-phase kernel estimation for robust motion deblurring," in Computer Vision - ECCV 2010, ser. LNCS, K. Daniilidis, P. Maragos, and N. Paragios, Eds. Springer Berlin - Heidelberg, 2010, vol. 6311, pp. 157-170.

[6] B. Zitová and J. Flusser, "Image registration methods: a survey," Image Vision Comput, vol. 11, no. 21, pp. 977-1000, 2003.

[7] C. Stewart, C.-L. Tsai, and B. Roysam, "The dual-bootstrap iterative closest point algorithm with application to retinal image registration," IEEE T Med Imaging, vol. 22, no. 11, pp. 1379-1394, 2003.

[8] M. Foracchia, E. Grisan, and A. Ruggeri, "Luminosity and contrast normalization in retinal images," Med Image Anal, vol. 9, no. 3, pp. 179-190, 2005.

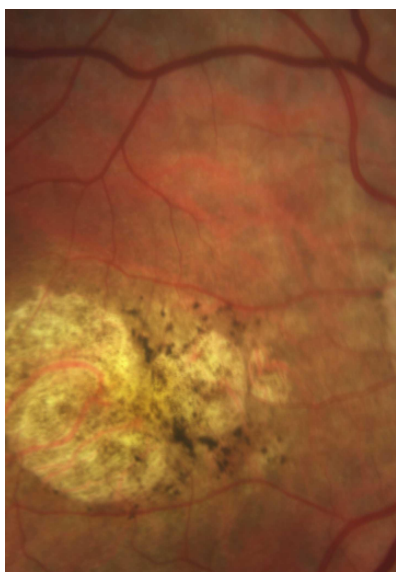

(a) Original $1\left(z_{1}\right)$

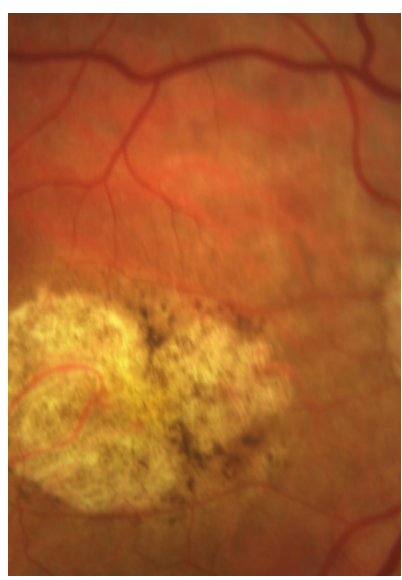

(c) Original $2\left(z_{2}\right)$

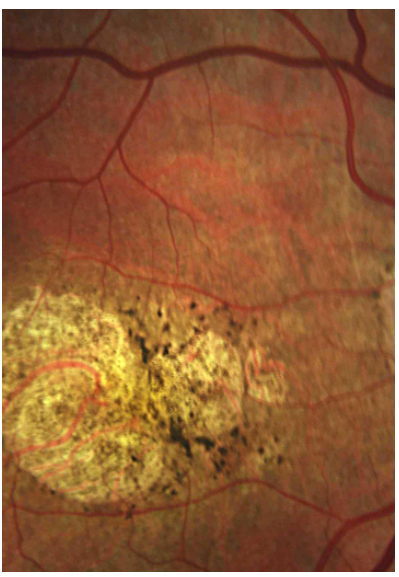

(b) Restored $1\left(u_{1}\right)$

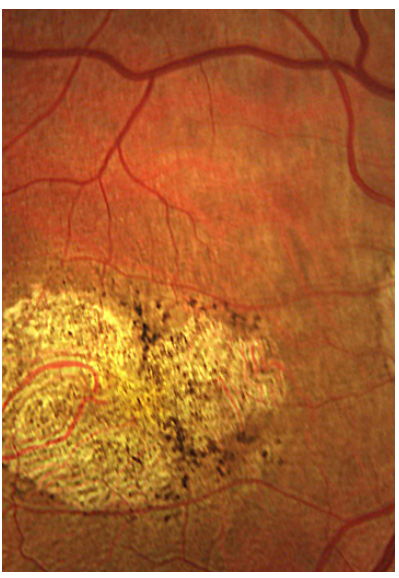

(d) Restored $2\left(u_{2}\right)$
Fig. 5. Original and restored color retinal images. The enhancement is much more noticeable in color.

[9] A. G. Marrugo and M. S. Millan, "Retinal image analysis: preprocessing and feature extraction," Journal of Physics: Conference Series, vol. 274 no. 1, p. 012039, 2011.

[10] N. Otsu, "A threshold selection method from gray-level histograms," IEEE T Systems, Man and Cybernetics, vol. 9, no. 1, pp. 62-66, 1979.

[11] F. Sroubek and J. Flusser, "Multichannel blind deconvolution of spatially misaligned images," IEEE T Image Process, vol. 14, no. 7, pp. 874-883, 2005.

[12] F. Šroubek, J. Flusser, and M. Šorel, "Superresolution and blind deconvolution of video," in Proc. IEEE Conf. Computer Vision and Pattern Recognition, 2008, pp. 1-4.

[13] A. Chambolle and P. Lions, "Image recovery via total variation minimization and related problems," Numer. Math., vol. 76, no. 2, pp. 167 $188,1997$. 